\title{
RELATIVE PARTITION FUNCTION OF COULOMB PLUS DELTA INTERACTION
}

\author{
SERGIO ALBEVERIO, CLAUDIO CACCIAPUOTI, AND MAURO SPREAFICO
}

\begin{abstract}
The relative partition function and the relative zeta function of the perturbation of the Laplace operator by a Coulomb potential plus a point interaction centered in the origin is discussed. Applications to the study of the Casimir effect are indicated.
\end{abstract}

Dedicated to Pavel Exner

\section{INTRODUCTION}

The present paper discusses a problem related to three main areas of investigations, in mathematics and physics: the theory of quantum fields (in particular thermal fields), the study of determinants of elliptic (pseudo differential) operators, and the study of singular perturbations of linear operators. The problem providing the link between these areas originated with a theoretical investigation by H. B. G. Casimir [20] who predicted the possibility of an effect, called "Casimir effect", of attraction of parallel conducting plates in vacuum due to the presence of fluctuations in the vacuum energy of the electromagnetic quantum field.

Since the experimental confirmation of this effect by Spaarnay [67], about ten years after the work of Casimir, both theoretical and experimental studies of "Casimir like effects" have received a lot of attention. In particular the temperature corrections where first discussed by M. Fierz [35] and J. Mehra [51, we refer to the monograph [12] for more references and details on the effects of temperature. On the other hand, its dependence on the geometry of the plates and the medium (even attractiveness can become repulsion according to changing geometry) has been discussed in several publications, see, e.g., the books [12, 19, 29, 30, 52, 54, the survey papers [11, 61], and, e.g., [10, 14, 15, 18, 21, 23, 25, 26, 27, 28, 31, 49, 56, 59, 62, 64.

2010 Mathematics Subject Classification. Primary 58J50, 11M36, 81Q80; Secondary $81 \mathrm{~T} 55$.

Key words and phrases. Relative zeta function, relative partition function, relative spectral measures, Coulomb interaction, point interactions, zeta regularization, finite temperature quantum fields, Casimir effect, asymptotic expansions. 
The physical discussion of the Casimir effect is also related to the one of the Van der Waals forces between molecules, see [54]. It has also many relations to condensed matter physics, hadronic physics, cosmology, and nanotechnology, see, e.g., the references in [11, 12, 29, 30, 52, 54, 61].

Theoretically the Casimir effect arises when computing the difference between two infinite quantities, namely the vacuum energy of a quantum field with or without a certain "boundary condition". More generally it is a phenomenon related to the difference of two Green's functions associated with hyperbolic or elliptic operators. Such problems are also of interest in geometric analysis, particularly since the work by W. Müller [55, and M. Spreafico and S. Zerbini 72. The latter works are related to the introduction by Ray and Singer [63] of a definition of determinants for elliptic operators on manifolds via a zeta-function renormalization (see also, e.g., [50, 57]). By this procedure one can define $\log (\operatorname{det} A)^{-\frac{1}{2}}$, for $A$ self adjoint, positive, in some Hilbert space, via the analytic continuation at $s=\frac{1}{2}$ of the zeta-function associated with $A$, defined for Re $s$ sufficiently large as

$$
\zeta(s ; A):=\sum_{\lambda \in \sigma^{+}(A)} \lambda^{-s}
$$

$\sigma^{+}(A)$ being the positive part of the spectrum of $A$. Setting

$$
Z:=(\operatorname{det} A)^{-\frac{1}{2}},
$$

one has the definition of the "partition function"

$$
\text { " } Z=\int_{\Phi} e^{-S(\varphi)} d \varphi ",
$$

$S(\varphi):=(\varphi, A \varphi)$, associated with a (Euclidean) quantum field with covariance operator given by the inverse of $A$ ( $\varphi$ is the field, $\Phi$ the space of "fields configurations").

In turn, it is well known that partitions functions $Z$ arise as normalizations in heuristic Euclidean path integrals

$$
\text { " } Z^{-1} \int_{\Phi} e^{-S(\varphi)} f(\varphi) d \varphi ",
$$

$f$ being complex valued functions (related to "observables"), see, e.g., [1, 73].

On the other hand it was pointed out by Hawking [43] and, independently, Figari, Høegh-Krohn, and Nappi [36], that there is a strict relation between Euclidean vacuum states in de Sitter spaces of fixed curvature and temperature states of Euclidean states. Hawking used the Ray-Singer definition of a partition function related to $A$ to compute physical quantities of the Euclidean model. For wide-ranging extensions of these connections see, e.g., [6, 7, 33, 34, 37, 38, 39, 53, 55, 68, 70]. 
Another application of the zeta function is in the computation of the high temperature asymptotics of several thermodynamic functions such as the Helmholtz free energy, internal energy, and entropy, see, e.g., [13] and references therein.

As pointed out in [55] and [69, 70, 71], considering the relative zetafunction of a pair of elliptic operators $A, A_{0}$, leads to define, via a relative zeta-function, a relative determinant including $A$ and $A_{0}$ and a Casimir effect can be discussed relatively to the pair $\left(A, A_{0}\right)$. In fact, the strength of the Casimir effect is expressed by the derivative of the relative zeta-function at 0 . These considerations are also related to the study of relative traces of semigroups resp. resolvents associated with pairs of operators. The study of such relative traces has its origins in quantum statistical mechanics [8].

The case where $A_{0}$ is the Laplace-Beltrami operator on $S^{1} \times \mathbb{R}^{3}$, and $A$ is a point perturbation of $A_{0}$ has been discussed in details in 72 and 3 . For the extended study of point interactions on $\mathbb{R}^{d}, d=1,2,3$, see [1, 4, 5]. The case where $\mathbb{R}^{d}$ is replaced by a Riemannian manifold occurs particularly in [24] (who points out its possible relevance in number theory), see also [22, 34, 48].

For further particular studies of point interactions in relation with the Casimir effect see [2, 4, 14, 15, 40, 42, 52, 45, 60, 65, 66.

Particularly close to our work is the result in [3] where $A_{0}$ is the half space $x^{3}>0$ in $\mathbb{R}^{3}$ and $A$ is taken to be the sum of two point interactions located at $\left(a_{1}, a_{2}, a_{3}\right)$ and $\left(a_{1}, a_{2},-a_{3}\right), a_{1}, a_{2} \in \mathbb{R}, a_{3} \in \mathbb{R}_{+}$. The relative trace of the resolvents was computed at values of the spectral parameter $\lambda$ such that $\operatorname{Im} \sqrt{\lambda}>0$, and the spectral measure was constructed. Moreover the asymptotics for small and large values of the spectral parameter was found. Furthermore the relative zeta-function and its derivative at 0 has been computed and related to the Casimir effect [3].

The present paper extends this kind of relations to the case of the pair $\left(A, A_{0}\right)$, where $A_{0}$ is the operator $-\Delta$ with a Coulomb interaction at the origin acting in $L^{2}\left(\mathbb{R}^{3}\right)$, and $A$ is a perturbation of $A_{0}$ obtained by adding a point interaction at the origin. The construction of $A_{0}$ and $A$ is based on [4], Ch I.2. In order to define and study the relative partition function we use explicit formulae for the integrals of the Whittaker's functions which enter the explicit expression of the resolvent of $-\Delta$ with a Coulomb interaction.

Such explicit formulae do not exist in the situation where the point interaction is not centered at the origin. In this situation an alternative approach would be to use series expansions to compute the integrals. It turns out that this idea does not seem feasible due to the slow decay of the Coulomb interaction at infinity. On the other hand, the case of potentials with faster decay at infinity should be treatable in this way, replacing the explicit formulae by methods of regular perturbations theory. 
The structure of the paper is as follows. In Section 2 we recall the general definition of the relative partition function associated to a pair of non-negative self-adjoint operators and its relation with the relative zeta function. In Section 3 we study the perturbation of the Laplacian by a Coulomb and a delta potential centered at the origin. In Section 4 we study the associated relative partition function of the Coulomb plus delta interaction.

\section{Relative PaRtition FunCtion ASSOCiATEd to A PAIR OF NON-NEGATIVE SELF ADJOINT OPERATORS}

This section presents a generalization of the method introduced in 72 ] to study the analytic properties of the relative zeta function associated to a pair of operators $\left(A, A_{0}\right)$ as described below (see also [55]). We assume here that logarithmic terms appear in the expansion of the relative trace, and this will produce a double pole in the relative zeta function, and in turn a simple pole in the relative partition function.

2.1. Relative zeta function. We denote by $R(\lambda ; A) \equiv(\lambda-A)^{-1}$ the resolvent of a linear operator $A$. $\lambda$ is in the resolvent set, $\rho(A)$, of $A$, a subset of $\mathbb{C}$. The relative zeta function $\zeta\left(s ; A, A_{0}\right)$ for a pair of non negative self adjoint operators $\left(A, A_{0}\right)$ is defined when the relative resolvent $R(\lambda ; A)-R\left(\lambda ; A_{0}\right)$ is of trace class and some conditions on the asymptotic expansions of the trace of the relative resolvent $r\left(\lambda ; A, A_{0}\right)$ are satisfied, as in Section 2 of [72. These conditions imply that similar conditions on the trace of the relative heat operator $\operatorname{Tr}\left(\mathrm{e}^{-t A}-\mathrm{e}^{-t A_{0}}\right)$ are satisfied, according to Section 2 of [55]. The conditions in [72] on the asymptotic expansions ensure that the relative zeta function is regular at $s=0$. In the present work we consider a wider class of pairs, and we admit a more general type of asymptotic expansions, as follows. Let $\mathcal{H}$ be a separable Hilbert space, and let $A$ and $A_{0}$ be two self adjoint non negative linear operators in $\mathcal{H}$. Suppose that $\mathrm{SpA}=\mathrm{Sp}_{\mathrm{c}} \mathrm{A}$, is purely continuous, and assume both 0 and $\infty$ are accumulation points of SpA.

Then, by a standard argument (see for example the proof of the corresponding result in [72]), we prove Lemma 2.1 below.

Let us recall first the definition of asymptotic expansion. If $f(\lambda)$ is a complex valued function, we write $f(\lambda) \sim \sum_{n=0}^{\infty} a_{n} \lambda^{n}, a_{n} \in \mathbb{C}, \lambda \rightarrow 0$, if for any $N \in \mathbb{N}_{0}$ one has $\frac{f(\lambda)-\sum_{n=0}^{N} a_{n} \lambda^{n}}{\lambda^{N}} \rightarrow 0$ as $\lambda \rightarrow 0$, and we say that $f$ has the asymptotic expansion $\sum_{n=0}^{\infty} a_{n} \lambda^{n}$. Then the following result holds true:

Lemma 2.1. Let $\left(A, A_{0}\right)$ be a pair of non negative self adjoint operators as above satisfying the following conditions: 
(B.1) the operator $R(\lambda ; A)-R\left(\lambda ; A_{0}\right)$ is of trace class for all $\lambda \in \rho(A) \cap$ $\rho\left(A_{0}\right)$;

(B.2) as $\lambda \rightarrow \infty$ in $\rho(A) \cap \rho\left(A_{0}\right)$, there exists an asymptotic expansion of the form:

$$
\operatorname{Tr}\left(R(\lambda ; A)-R\left(\lambda ; A_{0}\right)\right) \sim \sum_{j=0}^{\infty} \sum_{k=0}^{K_{j}} a_{j, k}(-\lambda)^{\alpha_{j}} \log ^{k}(-\lambda),
$$

where $a_{j, k} \in \mathbb{C},-\infty<\cdots<\alpha_{1}<\alpha_{0}, \alpha_{j} \rightarrow-\infty$, for large $j$;

(B.3) as $\lambda \rightarrow 0$, there exists an asymptotic expansion of the form

$$
\operatorname{Tr}\left(R(\lambda ; A)-R\left(\lambda ; A_{0}\right)\right) \sim \sum_{j=0}^{\infty} b_{j}(-\lambda)^{\beta_{j}}
$$

where $b_{j} \in \mathbb{C},-1 \leq \beta_{0}<\beta_{1}<\ldots$, and $\beta_{j} \rightarrow+\infty$, for large $j$,

(C) $\alpha_{0}<\beta_{0}$.

Then the relative zeta function is defined by

$$
\zeta\left(s ; A, A_{0}\right)=\frac{1}{\Gamma(s)} \int_{0}^{\infty} t^{s-1} \operatorname{Tr}\left(\mathrm{e}^{-t A}-\mathrm{e}^{-t A_{0}}\right) d t,
$$

when $\alpha_{0}+1<\operatorname{Re}(s)<\beta_{0}+1$, and by analytic continuation elsewhere. Here $\Gamma$ is the classical Gamma function and

$$
\operatorname{Tr}\left(\mathrm{e}^{-t A}-\mathrm{e}^{-t A_{0}}\right)=\frac{1}{2 \pi i} \int_{\Lambda} \mathrm{e}^{-\lambda t} \operatorname{Tr}\left(R(\lambda ; A)-R\left(\lambda ; A_{0}\right)\right) d \lambda,
$$

where $\Lambda$ is some contour of Hankel type (see, e.g., [32, 70]). The analytic extension of $\zeta\left(s ; A, A_{0}\right)$ is regular except for possible simple poles at $s=\beta_{j}$ and possible further poles at $s=\alpha_{j}$.

Note that the poles of the relative zeta function at $s=\alpha_{j}$ can be of higher orders, differently from the case investigated in [72].

Introducing the relative spectral measure, we have the following useful representation of the relative zeta function.

Lemma 2.2. Let $\left(A, A_{0}\right)$ be a pair of non negative self adjoint operators as above satisfying conditions (B.1)-(B.3), and (C) of Lemma 2.1. Then,

$$
\zeta\left(s ; A, A_{0}\right)=\int_{0}^{\infty} v^{-2 s} e\left(v ; A, A_{0}\right) d v,
$$

where the relative spectral measure is defined by

$$
\begin{aligned}
& e\left(v ; A, A_{0}\right)=\frac{v}{\pi i} \lim _{\epsilon \rightarrow 0^{+}}\left(r\left(v^{2} \mathrm{e}^{2 i \pi-i \epsilon} ; A, A_{0}\right)-r\left(v^{2} \mathrm{e}^{i \epsilon} ; A, A_{0}\right)\right) \quad v \geq 0, \\
& r\left(\lambda ; A, A_{0}\right)=\operatorname{Tr}\left(R(\lambda ; A)-R\left(\lambda ; A_{0}\right)\right) \quad \lambda \in \rho(A) \cap \rho\left(A_{0}\right) .
\end{aligned}
$$


The integral, the limit and the trace exist.

Proof. Since $\left(A, A_{0}\right)$ satisfies (B.1)-(B.3), we can write

$$
\operatorname{Tr}\left(\mathrm{e}^{-t A}-\mathrm{e}^{-t A_{0}}\right)=\frac{1}{2 \pi i} \int_{\Lambda} \mathrm{e}^{-\lambda t} \operatorname{Tr}\left(R(\lambda ; A)-R\left(\lambda ; A_{0}\right)\right) d \lambda .
$$

Changing the spectral variable $\lambda$ to $k=\lambda^{\frac{1}{2}}$, with the principal value of the square root, i.e. with $0<\arg k<\pi$, we get

$$
\operatorname{Tr}\left(\mathrm{e}^{-t A}-\mathrm{e}^{-t A_{0}}\right)=\frac{1}{\pi i} \int_{\gamma} \mathrm{e}^{-k^{2} t} \operatorname{Tr}\left(R\left(k^{2} ; A\right)-R\left(k^{2} ; A_{0}\right)\right) k d k,
$$

where $\gamma$ is the line $k=-i c$, for some $c>0$. Writing $k=v \mathrm{e}^{i \theta}, 0 \leq \theta<2 \pi$, and $r\left(\lambda ; A, A_{0}\right)=\operatorname{Tr}\left(R(\lambda ; A)-R\left(\lambda ; A_{0}\right)\right)$, a standard computation leads to

$$
\begin{aligned}
\operatorname{Tr}\left(\mathrm{e}^{-t A}-\mathrm{e}^{-t A_{0}}\right) & =\int_{0}^{\infty} \mathrm{e}^{-v^{2} t} e\left(v ; A, A_{0}\right) d v, \\
\zeta\left(s ; A, A_{0}\right) & =\int_{0}^{\infty} v^{-2 s} e\left(v ; A, A_{0}\right) d v .
\end{aligned}
$$

Remark 2.3. The relative spectral measure is discussed in general, e.g., in [55. It is expressed by (2.2) in terms of $r\left(\lambda ; A, A_{0}\right)$ which is the Laplace transform of $\operatorname{Tr}\left(\mathrm{e}^{-t A}-\mathrm{e}^{-t A_{0}}\right)$, which in turn is simply related to the spectral shift function (see Eq. (0.6) in [55]). The derivative of the latter is essentially the density of states used, e.g., in 58 in connection with the Casimir effect, and going back to the original work by M.G. Krein and M.Sh. Birman [9, 46, 47].

It is clear by construction that the analytic properties of the relative zeta function are determined by the asymptotic expansions required in conditions (B.1) and (B.2). More precisely, such conditions imply similar conditions on the expansion of the relative spectral measure, and hence on the analytic structure of the relative zeta function. This is in the next lemmas.

Lemma 2.4. Let $\left(A, A_{0}\right)$ be a pair of non negative self adjoint operators as in Lemma 2.2. Then the relative spectral measure $e\left(v ; A, A_{0}\right)$ has the following asymptotic expansions. For small $v \geq 0$ :

$$
e\left(v ; A, A_{0}\right) \sim \sum_{j=0}^{\infty} c_{j} v^{2 \beta_{j}+1},
$$

where

$$
c_{j}=-\frac{2 b_{j} \sin \pi \beta_{j}}{\pi}
$$


and the $\beta_{j}$ and the $b_{j}$ are the numbers appearing in condition (B3) of Lemma 2.1; for large $v \geq 0$ and $j \in \mathbb{N}_{0}$ :

$$
\begin{aligned}
e\left(v ; A, A_{0}\right) & \sim \sum_{j=0}^{\infty} \sum_{h=0}^{H_{j}} e_{j, h} v^{2 \alpha_{j}+1} \log ^{h} v \\
& \sim \sum_{j=0}^{\infty} \sum_{k=0}^{K_{j}} \sum_{h=0}^{k} e_{j, k, h} v^{2 \alpha_{j}+1} \log ^{h} v^{2},
\end{aligned}
$$

where

$$
e_{j, k, h}=-a_{j, k}(\pi i)^{k-h-1}\left(\begin{array}{l}
k \\
h
\end{array}\right)\left(\mathrm{e}^{i \alpha_{j} \pi}-(-1)^{k-h} \mathrm{e}^{-i \alpha_{j} \pi}\right),
$$

and the $a_{j, k}, \alpha_{j}$, and $K_{j}$ are the numbers appearing in condition (B2) of Lemma 2.1. The coefficients $e_{j, h}$ can be expressed in terms of the coefficients $e_{j, k, h}$.

Proof. Note that the cut $(0, \infty)$ in the complex $\lambda$-plane corresponds to the cut $(-\infty, 0)$ in the complex $-\lambda$-plane. Thus $-\lambda=x \mathrm{e}^{i \theta}$, with $-\pi \theta<\pi$, and $\theta=0$ corresponds to positive real values of $-\lambda$.

Thus, inserting the expansion (B3) for small $\lambda$ in the definition of the relative spectral measure, equation (2.1), we obtain, for small $v$,

$$
e\left(v ; A, A_{0}\right) \sim-\frac{v}{i \pi} \lim _{\epsilon \rightarrow 0^{+}} \sum_{j=0}^{\infty} b_{j} v^{2 \beta_{j}}\left(\mathrm{e}^{(\pi i-i \epsilon) \beta_{j}}-\mathrm{e}^{(-\pi i+i \epsilon) \beta_{j}}\right),
$$

and the first part of the statement follows. For the expansion for large $v$, we insert (B2) into the definition of the relative spectral measure. This gives, for large $v$,

$$
\begin{aligned}
& e\left(v ; A, A_{0}\right) \\
& \sim-\frac{v}{i \pi} \lim _{\epsilon \rightarrow 0^{+}} \sum_{j=0}^{\infty} \sum_{k=0}^{K_{j}} a_{j, k} v^{2 \alpha_{j}} \\
& \times\left(\mathrm{e}^{(\pi i-i \epsilon) \alpha_{j}}\left(\log v^{2}+\pi i-i \epsilon\right)^{k}-\mathrm{e}^{(-\pi i+i \epsilon) \alpha_{j}}\left(\log v^{2}-\pi i+i \epsilon\right)^{k}\right), \\
& e\left(v ; A, A_{0}\right) \\
& \sim-\sum_{j=0}^{\infty} v^{2 \alpha_{j}+1} \sum_{k=0}^{K_{j}} \frac{a_{j, k}}{i \pi} \\
& \times\left(\mathrm{e}^{\pi i \alpha_{j}} \sum_{h=0}^{k}\left(\begin{array}{l}
k \\
h
\end{array}\right)(i \pi)^{k-h} \log ^{k} v^{2}-\mathrm{e}^{-\pi i \alpha_{j}} \sum_{h=0}^{k}\left(\begin{array}{l}
k \\
h
\end{array}\right)(-i \pi)^{k-h} \log ^{k} v^{2}\right),
\end{aligned}
$$

and the thesis follows. 
Remark 2.5. We give more details on the first coefficients that are more relevant in the present work. Direct calculation gives

$$
\begin{aligned}
e_{j, 0} & =e_{j, 0,0}+2 \sum_{k=1}^{K_{j}} e_{j, k, 0}=-\sum_{k=0}^{K_{j}} a_{j, k}(\pi i)^{k-1}\left(\mathrm{e}^{i \alpha_{j} \pi}-(-1)^{k} \mathrm{e}^{-i \alpha_{j} \pi}\right), \\
e_{j, 0,0} & =-\frac{2 \sin \pi \alpha_{j}}{\pi} a_{j, 0} .
\end{aligned}
$$

2.2. Relative partition function. Let $W$ be a smooth Riemannian manifold of dimension $n$, and consider the product $X=S_{\frac{\beta}{2 \pi}}^{1} \times W$, where $S_{r}^{1}$ is the circle of radius $r, \beta>0$. Let $\xi$ be a complex line bundle over $X$, and $L$ a self adjoint non negative linear operator on the Hilbert space $\mathcal{H}(W)$ of the $L^{2}$ sections of the restriction of $\xi$ onto $W$, with respect to some fixed metric $g$ on $W$. Let $L$ be the self adjoint non negative operator $L=-\partial_{u}^{2}+A$, on the Hilbert space $\mathcal{H}(X)$ of the $L^{2}$ sections of $\xi$, with respect to the product metric $d u^{2} \oplus g$ on $X$, and with periodic boundary conditions on the circle. Assume that there exists a second operator $A_{0}$ defined on $\mathcal{H}(W)$, such that the pair $\left(A, A_{0}\right)$ satisfies the assumptions (B.1)-(B.3) of Lemma 2.1. Then, by a proof similar to the one of Lemma 2.1 of 72 , it is possible to show that there exists a second operator $L_{0}$ defined in $\mathcal{H}(X)$, such that the pair $\left(L, L_{0}\right)$ satisfies those assumptions too. Under these requirements, we define the regularized relative zeta partition function of the model described by the pair of operators $\left(L, L_{0}\right)$ by

$$
\log Z_{\mathrm{R}}=\frac{1}{2} \operatorname{Res}_{s=0} \zeta^{\prime}\left(s ; L, L_{0}\right)-\frac{1}{2} \operatorname{Res}_{s=0} \zeta\left(s ; L, L_{0}\right) \log \ell^{2},
$$

where $\ell$ is some renormalization constant (introduced by Hawking [43], see also, e.g., [53], in connection with the scaling behavior in path integrals in curved spaces), and we have the following result, in which $\log Z_{R}$ is essentially expressed in terms of the relative Dedekind eta function $\eta\left(\beta ; A, A_{0}\right)$.

Proposition 2.6. Let $A$ be a non negative self adjoint operator on $W$, and $L=-\partial_{u}^{2}+A$, on $S_{\frac{\beta}{2 \pi}}^{1} \times W$ as defined above. Assume there exists an operator $A_{0}$ such that the pair $\left(A, A_{0}\right)$ satisfies conditions (B.1)-(B.3) of Lemma 2.1. Then, the relative zeta function $\zeta\left(s ; L, L_{0}\right)$ (defined analogously to the one 
given in Lemma 2.1) has a simple pole at $s=0$ with residua:

$$
\begin{aligned}
\operatorname{Res}_{s=0} \zeta\left(s ; L, L_{0}\right)= & -\beta \operatorname{Res}_{s=-\frac{1}{2}} \zeta\left(s ; A, A_{0}\right), \\
\operatorname{Res}_{s=0} \zeta\left(s ; L, L_{0}\right)= & -\beta \operatorname{Res}_{s=-\frac{1}{2}} \zeta\left(s ; A, A_{0}\right)-2 \beta(1-\log 2) \operatorname{Res}_{s=-\frac{1}{2}} \zeta\left(s ; A, A_{0}\right), \\
\operatorname{Res}_{s=0} \zeta^{\prime}\left(s ; L, L_{0}\right)= & -\beta \operatorname{Res}_{s=-\frac{1}{2}} \zeta\left(s ; A, A_{0}\right)-2 \beta(1-\log 2) \operatorname{Res}_{s=-\frac{1}{2}} \zeta\left(s ; A, A_{0}\right) \\
& -\beta\left(2+\frac{\pi^{2}}{6}+2(1-\log 2)^{2}\right) \operatorname{Res}_{s=-\frac{1}{2}} \zeta\left(s ; A, A_{0}\right) \\
& -2 \log \eta\left(\beta ; A, A_{0}\right),
\end{aligned}
$$

where $L_{0}=-\partial_{u}^{2}+A_{0}$, and the relative Dedekind eta function is defined by

$$
\log \eta\left(\tau ; A, A_{0}\right)=\int_{0}^{\infty} \log \left(1-\mathrm{e}^{-\tau v}\right) e\left(v ; A, A_{0}\right) d v, \quad \tau>0 .
$$
$\operatorname{Res}_{\substack{\mathrm{k} \\ s=s_{0}}} \zeta(s)$ is understood as the coefficient of the term $\left(s-s_{0}\right)^{-k}$ in the Laurent expansion of $\zeta(s)$ around $s=s_{0}$.

The residua and the integral are finite.

Proof. Since $\left(A, A_{0}\right)$ satisfies (B.1)-(B.3), the $\left(L, L_{0}\right)$ relative zeta function $\zeta\left(s ; L, L_{0}\right)$ is defined by

$$
\zeta\left(s ; L, L_{0}\right)=\frac{1}{\Gamma(s)} \int_{0}^{\infty} t^{s-1} \operatorname{Tr}\left(\mathrm{e}^{-t L}-\mathrm{e}^{-t L_{0}}\right) d t,
$$

when $\alpha_{0}+1<\operatorname{Re}(s)<\beta_{0}+1$ (with $\alpha_{0}$ and $\beta_{0}$ as in Lemma 2.1). Since (see for example Lemma 2.2 of [72])

$$
\operatorname{Tr}\left(\mathrm{e}^{-L t}-\mathrm{e}^{-L_{0} t}\right)=\sum_{n \in \mathbb{Z}} \mathrm{e}^{-\frac{n^{2}}{r^{2}} t} \operatorname{Tr}\left(\mathrm{e}^{-t A}-\mathrm{e}^{-t A_{0}}\right),
$$

where $r=\beta /(2 \pi)$ and $t>0$. Using the Jacobi summation formula and dominated convergence to exchange summation and integration we obtain

$$
\begin{aligned}
\zeta\left(s ; L, L_{0}\right)= & \frac{1}{\Gamma(s)} \int_{0}^{\infty} t^{s-1} \sum_{n \in \mathbb{Z}} \mathrm{e}^{-\frac{n^{2}}{r^{2}} t} \operatorname{Tr}\left(\mathrm{e}^{-t A}-\mathrm{e}^{-t A_{0}}\right) d t \\
= & \frac{\sqrt{\pi} r}{\Gamma(s)} \int_{0}^{\infty} t^{s-\frac{1}{2}-1} \operatorname{Tr}\left(\mathrm{e}^{-t A}-\mathrm{e}^{-t A_{0}}\right) d t \\
& +\frac{2 \sqrt{\pi} r}{\Gamma(s)} \int_{0}^{\infty} t^{s-\frac{1}{2}-1} \sum_{n=1}^{\infty} \mathrm{e}^{-\frac{\pi^{2} r^{2} n^{2}}{t}} \operatorname{Tr}\left(\mathrm{e}^{-t A}-\mathrm{e}^{-t A_{0}}\right) d t \\
= & z_{1}(s)+z_{2}(s),
\end{aligned}
$$


with

$$
\begin{aligned}
& z_{1}(s):=\frac{\sqrt{\pi} r}{\Gamma(s)} \Gamma\left(s-\frac{1}{2}\right) \zeta\left(s-\frac{1}{2} ; A, A_{0}\right) \\
& z_{2}(s):=\frac{2 \sqrt{\pi} r}{\Gamma(s)} \sum_{n=1}^{\infty} \int_{0}^{\infty} t^{s-\frac{1}{2}-1} \mathrm{e}^{-\frac{\pi^{2} r^{2} n^{2}}{t}} \operatorname{Tr}\left(\mathrm{e}^{-t A}-\mathrm{e}^{-t A_{0}}\right) d t .
\end{aligned}
$$

The first term, $z_{1}(s)$, can be expanded near $s=0$, and this gives the result stated, by Lemma 2.1. By Lemma 2.2, the second term $z_{2}(s)$ is

$$
z_{2}(s)=\frac{2 \sqrt{\pi} r}{\Gamma(s)} \sum_{n=1}^{\infty} \int_{0}^{\infty} t^{s-\frac{1}{2}-1} \mathrm{e}^{-\frac{\pi^{2} n^{2} r^{2}}{t}} \int_{0}^{\infty} \mathrm{e}^{-v^{2} t} e\left(v ; A, A_{0}\right) d v d t
$$

and we can do the $t$ integral using for example [41, 3.471.9]. We obtain

$$
z_{2}(s)=\frac{4 \sqrt{\pi} r}{\Gamma(s)} \sum_{n=1}^{\infty} \int_{0}^{\infty}\left(\frac{\pi n r}{v}\right)^{s-\frac{1}{2}} K_{s-\frac{1}{2}}(2 \pi n r v) e\left(v ; A, A_{0}\right) d v
$$

Since the Bessel function $K_{s-\frac{1}{2}}(2 \pi n r v)$ is analytic in its parameter, regular at $-\frac{1}{2}$, and $K_{-\frac{1}{2}}(z)=\sqrt{\frac{\pi}{2 z}} \mathrm{e}^{-z}$, equation (2.4) gives the formula for the analytic extension of the relative zeta function $\zeta\left(s ; H, H_{0}\right)$ near $s=0$. We obtain

$$
z_{2}(0)=0, \quad z_{2}^{\prime}(0)=-2 \int_{0}^{\infty} \log \left(1-\mathrm{e}^{-2 \pi r v}\right) e\left(v ; L, L_{0}\right) d v
$$

and the integral converges by assumptions (B.2) and (B.3).

It is clear by the previous result that all information on the relative partition function comes from the analytic structure of the spatial relative spectral function $\zeta\left(s ; A, A_{0}\right)$ near $s=-\frac{1}{2}$. Such information is based on the asymptotic expansion assumed for the relative resolvent, and contained in the following lemma. 
Lemma 2.7. Let $\left(A, A_{0}\right)$ be a pair of non negative self adjoint operators as in Lemma 2.2. Then, the relative zeta function $\zeta\left(s ; A, A_{0}\right)$ extends analytically to the following meromorphic function in a neighborhood of $s=-\frac{1}{2}$ :

$$
\begin{aligned}
\zeta\left(s ; A, A_{0}\right)= & \frac{1}{2} \sum_{j=0}^{J_{0}-1} \frac{c_{j}}{\beta_{j}+1-s}+\sum_{j=0}^{J_{\infty}-1} \sum_{h=0}^{H_{j}} \frac{(-1)^{h+1} e_{j, h}}{2^{h+1}\left(\alpha_{j}+1-s\right)^{h+1}} \\
& +\int_{0}^{1} v^{-2 s}\left(e\left(s ; A, A_{0}\right)-\sum_{j=0}^{J_{0}} c_{j} v^{2 \beta_{j}+1}\right) d v \\
& +\int_{1}^{\infty} v^{-2 s}\left(e\left(s ; A, A_{0}\right)-\sum_{j=0}^{J_{\infty}} \sum_{h=0}^{H_{j}} e_{j, h} v^{2 \alpha_{j}+1} \log ^{h} v\right) d v,
\end{aligned}
$$

where $J_{0}$ is the smallest integer such that $\beta_{J_{0}}>-\frac{3}{2}$, and $J_{\infty}$ is the largest integer such that $\alpha_{J_{\infty}}<-\frac{3}{2}$ (the $\alpha_{j}, \beta_{j}$ resp. $c_{j}, e_{j, h}, H_{j}$ are as in Lemma 2.1 resp. Lemma (2.2).

Proof. Set

$$
\zeta_{0}\left(s ; A, A_{0}\right)=\int_{0}^{1} v^{-2 s} e\left(v ; A, A_{0}\right) d v
$$

and

$$
\zeta_{\infty}\left(s ; A, A_{0}\right)=\int_{1}^{\infty} v^{-2 s} e\left(v ; A, A_{0}\right) d v
$$

then

$$
\zeta\left(s ; A, A_{0}\right)=\zeta_{0}\left(s ; A, A_{0}\right)+\zeta_{\infty}\left(s ; A, A_{0}\right) .
$$

Consider the expansion of $e\left(s ; A, A_{0}\right)$ for small $v$ given in Lemma 2.4 Let $J_{0}$ be the smallest integer such that $\beta_{J_{0}}>-\frac{3}{2}$, and write

$$
\zeta_{0}\left(s ; A, A_{0}\right)
$$

$$
=\int_{0}^{1} v^{-2 s}\left(\sum_{j=0}^{J_{0}} c_{j} v^{2 \beta_{j}+1}\right) d v+\int_{0}^{1} v^{-2 s}\left(e\left(s ; A, A_{0}\right)-\sum_{j=0}^{J_{0}} c_{j} v^{2 \beta_{j}+1}\right) d v .
$$

The last integral in equation (2.6) is convergent, while the first one can be computed explicitly. This gives the statement for $\zeta_{0}$, in the sense that $\zeta_{0}$ has a representation like in equation (2.5) . For $\zeta_{\infty}$ consider the expansion of $e\left(s ; A, A_{0}\right)$ for large $v$ given in Lemma 2.4, Let $J_{\infty}$ be the largest integer 
such that $\alpha_{J_{\infty}}<-\frac{3}{2}$, and write

$$
\begin{aligned}
\zeta_{\infty}\left(s ; A, A_{0}\right)= & \int_{1}^{\infty} v^{-2 s}\left(\sum_{j=0}^{J_{\infty}} \sum_{h=0}^{H_{j}} e_{j, h} v^{2 \alpha_{j}+1} \log ^{h} v\right) d v \\
& +\int_{1}^{\infty} v^{-2 s}\left(e\left(s ; A, A_{0}\right)-\sum_{j=0}^{J_{\infty}} \sum_{h=0}^{H_{j}} e_{j, h} v^{2 \alpha_{j}+1} \log ^{h} v\right) d v .
\end{aligned}
$$

The last integral in equation (2.7) is convergent, while the first one can be computed explicitly. This gives the statement for $\zeta_{\infty}$, in the sense that $\zeta_{\infty}$ has a representation like in equation (2.5). Putting together the representations of $\zeta_{0}$ and $\zeta_{\infty}$ concludes the proof.

Corollary 2.8. Let $\left(A, A_{0}\right)$ be a pair of non negative self adjoint operators as in Lemma 2.2. With the notation of that lemma,

$$
\begin{aligned}
& \operatorname{Res}_{2} \zeta\left(s ; A, A_{0}\right)= \frac{e_{a, 1}}{4}, \\
& \operatorname{Res}_{1} \zeta \\
& s=-\frac{1}{2} \\
& \operatorname{Res}_{0} \zeta\left(s ; A, A_{0}\right)= \frac{e_{a, 0}}{2}-\frac{c_{b}}{2}, \\
& s_{s=-\frac{1}{2}}+\int_{0}^{1} v\left(e\left(-\frac{1}{2} ; A, A_{0}\right)-\sum_{j=0}^{J_{0}} c_{j} v^{2 \beta_{j}+1}\right) d v \\
&+\int_{1}^{\infty} v\left(e\left(-\frac{1}{2} ; A, A_{0}\right)-\sum_{j=0}^{J_{\infty}} \sum_{h=0}^{H_{j}} e_{j, h} v^{2 \alpha_{j}+1} \log ^{h} v\right) d v,
\end{aligned}
$$

where in the lower limits of the sums a is the index in the sequence $\left\{\alpha_{j}\right\}$, such that $\alpha_{a}=-\frac{3}{2}$, and $b$ is the index in the sequence $\left\{\beta_{j}\right\}$, such that 


$$
\begin{aligned}
\beta_{b}=-\frac{3}{2}, \text { and } & \\
\operatorname{Res}_{s=0} \zeta\left(s ; L, L_{0}\right)= & -\frac{e_{a, 1}}{4} \beta, \\
\operatorname{Res}_{s=0} \zeta\left(s ; L, L_{0}\right)= & -\frac{1}{2}\left(c_{b}-e_{a, 0}-(1-\log 2) e_{a, 1}\right) \beta, \\
\operatorname{Res}_{s=0} \zeta^{\prime}\left(s ; L, L_{0}\right)= & -\beta\left(\frac{1}{2} \sum_{j=0, j \neq b}^{J_{0}} \frac{c_{j}}{\beta_{j}+3 / 2}+\sum_{j=0, j \neq a}^{J_{\infty}} \sum_{h=0}^{H_{j}} \frac{(-1)^{h+1} e_{j, h}}{2^{h+1}\left(\alpha_{j}+3 / 2\right)^{h+1}}\right. \\
& +\int_{0}^{1} v\left(e\left(-1 / 2 ; A, A_{0}\right)-\sum_{j=0}^{J_{0}} c_{j} v^{2 \beta_{j}+1}\right) d v \\
& \left.+\int_{1}^{\infty} v\left(e\left(-1 / 2 ; A, A_{0}\right)-\sum_{j=0}^{J_{\infty}} \sum_{h=0}^{H_{j}} e_{j, h} v^{2 \alpha_{j}+1} \log ^{h} v\right) d v\right) \\
& -\beta(1-\log 2)\left(e_{a, 0}-c_{b}\right) \\
& -\beta\left(\frac{1}{2}+\frac{\pi^{2}}{24}+\frac{(1-\log 2)^{2}}{2}\right) e_{a, 1} \\
& -2 \log \eta\left(\beta ; A, A_{0}\right) .
\end{aligned}
$$

Proof. This is a simple consequence of Lemma 2.7 and Prop. 2.6

\section{Coulomb potential plus delta interaction centered at the ORIGIN}

3.1. Preliminaries. Recall that we denote by $\rho(A)$ the resolvent set of $A$ and by $R(\lambda ; A)$ the resolvent operator $(\lambda I-A)^{-1}$, for $\lambda \in \rho(A)$. If $R(\lambda ; A)$ operates in $L^{2}\left(\mathbb{R}^{3}\right)$, we denote by $k(\lambda ; A)=k(\lambda ; A)(x, y)$ the integral kernel of $R(\lambda ; A), x, y \in \mathbb{R}^{3}$.

Let $H_{0}$ be the self-adjoint realization of the operator $-\Delta+\gamma /|x|$ in $L^{2}\left(\mathbb{R}^{3}\right)$, namely the Laplace operator plus a Coulomb potential centered at the origin in the three dimensional Euclidean space, with parameter $\gamma \in \mathbb{R}$. The kernel of the resolvent of $H_{0}$ is, see, e.g., [4, eq. (2.1.16)] and [16, 17,

$$
k\left(\lambda ; H_{0}\right)(x, y)=-\frac{\Gamma(1+\gamma / 2 \sqrt{-\lambda})}{4 \pi|x-y|} f_{\lambda}(x, y),
$$

where

$$
\begin{aligned}
f_{\lambda}(x, y)= & W_{-\frac{\gamma}{2 \sqrt{-\lambda}}, \frac{1}{2}}\left(\sqrt{-\lambda} x_{+}\right) M_{-\frac{\gamma}{2 \sqrt{-\lambda}}, \frac{1}{2}}^{\prime}\left(\sqrt{-\lambda} x_{-}\right) \\
& -W_{-\frac{\gamma}{2 \sqrt{-\lambda}}, \frac{1}{2}}^{\prime}\left(\sqrt{-\lambda} x_{+}\right) M_{-\frac{\gamma}{2 \sqrt{-\lambda}}, \frac{1}{2}}\left(\sqrt{-\lambda} x_{-}\right),
\end{aligned}
$$


with $\lambda \in \rho\left(H_{0}\right), \operatorname{Re} \sqrt{-\lambda}>0, x_{ \pm}=|x|+|y| \pm|x-y|$, and where $M_{\kappa, \mu}$ and $W_{\kappa, \mu}$ are Whittaker functions, see e.g. [41]. In the next proposition we recall some results on the spectrum of $H_{0}$, see e.g. [4, 44].

Proposition 3.1. For all $\gamma \in \mathbb{R}$ the essential spectrum of $H_{0}$ is purely absolutely continuous, moreover

$$
\sigma_{\text {ess }}\left(H_{0}\right)=\sigma_{a c}\left(H_{0}\right)=[0,+\infty) .
$$

If $\gamma \geq 0$ the point spectrum of $H_{0}$ is empty. If $\gamma<0$ the point spectrum of $H_{0}$ is

$$
\sigma_{p p}\left(H_{0}\right)=\left\{-\frac{\gamma^{2}}{4(n+1)^{2}}\right\}_{n=0}^{\infty} \quad \gamma<0 .
$$

Following [4, we introduced a perturbation of $H_{0}$, by adding a singular one center point interaction, also centered at the origin. For all $-\infty<\alpha \leq$ $\infty$ we denote by $H_{\alpha}$ the operator formally written as $-\Delta+\gamma /|x|+\alpha \delta_{0}$. The concrete operator is defined in Theorem 2.1.2 of [4, and the integral kernel of the resolvent of $H_{\alpha}$ is

$$
\begin{gathered}
k\left(\lambda ; H_{\alpha}\right)(x, y)=k\left(\lambda ; H_{0}\right)(x, y)-\frac{4 \pi}{4 \pi \alpha-\gamma F_{\gamma}(\gamma / 2 \sqrt{-\lambda})} g(\lambda ; x) g(\lambda ; y), \\
\lambda \in \rho\left(H_{\alpha}\right) \cap \rho\left(H_{0}\right), \operatorname{Re} \sqrt{-\lambda}>0, x, y \in \mathbb{R}^{3}
\end{gathered}
$$

with

$$
g(\lambda ; x):=\frac{\Gamma(1+\gamma / 2 \sqrt{-\lambda})}{4 \pi|x|} W_{-\gamma / 2 \sqrt{-\lambda}, 1 / 2}(2 \sqrt{-\lambda}|x|) \quad x \neq 0
$$

and

$$
F_{\gamma}(z):= \begin{cases}\psi(1+z)-\log (z)-\frac{1}{2 z}-\psi(1)-\psi(2), & \gamma>0 \\ \psi(1+z)-\log (-z)-\frac{1}{2 z}-\psi(1)-\psi(2), & \gamma<0 .\end{cases}
$$

Here $z \in \mathbb{C}$ and $\psi$ is the digamma function, i.e., $\psi(z)=\frac{d}{d z} \log \Gamma(z)$, see [41, 8.36]. We note that the function $F_{\gamma}(z)$ is indeed a function of $z$ and of $\operatorname{sgn}(\gamma)$ only.

In the following proposition we recall some results on the spectrum of the operator $H_{\alpha}$, see, e.g. [4, Th. 2.1.3].

Proposition 3.2. Let $-\infty<\alpha \leq \infty$. For all $\gamma \in \mathbb{R}$ the essential spectrum of $H_{\alpha}$ is purely absolutely continuous, moreover

$$
\sigma_{\text {ess }}\left(H_{\alpha}\right)=\sigma_{a c}\left(H_{\alpha}\right)=[0,+\infty) .
$$

The eigenvalues of $H_{\alpha}$ associated with the $s$-wave $(l=0)$ are given by the solutions of the equation

$$
4 \pi \alpha-\gamma F_{\gamma}(\gamma / 2 \sqrt{-E})=0, \quad E<0,
$$


where we set $\left.\gamma F_{\gamma}(\gamma / 2 \sqrt{-E})\right|_{\gamma=0}=\lim _{\gamma \rightarrow 0} \gamma F_{\gamma}(\gamma / 2 \sqrt{-E})=-\sqrt{-E}$.

If $\gamma \geq 0$ and $\alpha \geq-\gamma[\psi(1)+\psi(2)] / 4 \pi$, the equation (3.2) has no solutions, moreover the point spectrum of $H_{\alpha}$ is empty.

If $\gamma \geq 0$ and $\alpha<-\gamma[\psi(1)+\psi(2)] / 4 \pi$, the equation (3.2) has precisely one solution, and the operator $H_{\alpha}$ has precisely one negative eigenvalue.

If $\gamma<0$ the equation (3.2) has infinitely many solutions. Correspondingly there are infinitely many simple eigenvalues associated with the $s$-wave $(l=$ $0)$, moreover for $l \geq 1$ the eigenvalues of $H_{\alpha}$ are given by the usual Coulomb levels $E_{m}=-\gamma^{2} / 4 m^{2}, m \in \mathbb{N}, m \geq 2$.

Because of the results of the previous proposition, we proceed our analysis only in the case of repulsive Coulomb potential, namely for $\gamma \geq 0$.

3.2. Trace of the relative resolvent. We first note that for any $\lambda \in$ $\rho\left(H_{0}\right) \cap \rho\left(H_{\alpha}\right)$ the difference $\operatorname{Tr}\left(R\left(\lambda ; H_{\alpha}\right)-R\left(\lambda ; H_{0}\right)\right)$ is a rank one operator (see, e.g. [4]), then the trace of the relative resolvent of the pair $\left(H_{\alpha}, H_{0}\right)$ is well defined by

$$
r\left(\lambda ; H_{\alpha}, H_{0}\right)=\operatorname{Tr}\left(R\left(\lambda ; H_{\alpha}\right)-R\left(\lambda ; H_{0}\right)\right) .
$$

By equation (3.1) and by the definition of $g(\lambda, x)$ it follows that

$$
r\left(\lambda ; H_{\alpha}, H_{0}\right)=-\frac{\Gamma(1+\gamma / 2 \sqrt{-\lambda})^{2}}{4 \pi \alpha-\gamma F_{\gamma}(\gamma / 2 \sqrt{-\lambda})} \int_{0}^{\infty} W_{-\gamma / 2 \sqrt{-\lambda, 1 / 2}}^{2}(2 \sqrt{-\lambda}|x|) d|x|
$$

with $\operatorname{Re} \sqrt{-\lambda}>0$.

From [41, 7.625.4 and 9.302.1] and by using the identities $\Gamma(1+z)=z \Gamma(z)$ and $\Gamma(1-z) \Gamma(z)=\frac{\pi}{\sin (\pi z)}, z \in \mathbb{C} \backslash \mathbb{Z}$, we get for the integral in the latter equation the expression

$$
\begin{aligned}
& \int_{0}^{\infty} W_{-\gamma / 2 \sqrt{-\lambda}, 1 / 2}^{2}(2 \sqrt{-\lambda}|x|) d|x| \\
= & \frac{1}{2 \sqrt{-\lambda}} \frac{1}{\Gamma(1+\gamma / 2 \sqrt{-\lambda}) \Gamma(\gamma / 2 \sqrt{-\lambda})} \\
& \times \frac{1}{2 \pi i} \int_{L} \frac{(1-s) s}{(s+\gamma / 2 \sqrt{-\lambda})(s-1+\gamma / 2 \sqrt{-\lambda})} \frac{\pi^{2}}{\sin ^{2}(\pi s)} d s
\end{aligned}
$$

where $L$ is a path in $\mathbb{C}$ from $-\infty$ to $+\infty$ such that the set $\{1,2,3, \ldots\}$ is on the right of $L$ and the set $\{0,-1,-2, \ldots, 1-\gamma / 2 \sqrt{-\lambda},-\gamma / 2 \sqrt{-\lambda},-1-$ $\gamma / 2 \sqrt{-\lambda}, \ldots\}$ is on the left of $L$. We notice that for $\gamma>0$ one can choose $L=\left\{s=x_{0}+i y\right.$ with $\left.1-\gamma \operatorname{Re} \sqrt{-\lambda} / 2|\lambda|<x_{0}<1,-\infty<y<\infty\right\}$.

This gives

$$
r\left(\lambda ; H_{\alpha}, H_{0}\right)=-\frac{1}{4 \pi \alpha-\gamma F_{\gamma}(\gamma / 2 \sqrt{-\lambda})} \frac{1}{2 \sqrt{-\lambda}} I(\gamma / 2 \sqrt{-\lambda}),
$$


where

$$
I(z)=\frac{z}{2 \pi i} \int_{L} \frac{(1-s) s}{(s+z)(s-1+z)} \frac{\pi^{2}}{\sin ^{2}(\pi s)} d s,
$$

and we used again the identity $\Gamma(1+z)=z \Gamma(z), z \in \mathbb{C}$. In order to analyze the function $I(z)$ appearing in the formula for the relative trace of the resolvent we need the formulas in the following lemma.

Lemma 3.3. Let $L$ be the path $L=\left\{z=x_{0}+i y \mid 1-a<x_{0}<1,-\infty<\right.$ $y<\infty\}$, with $\operatorname{Re}(a)>0$, then

$$
\frac{1}{2 \pi i} \int_{L} \frac{\pi^{2}}{\sin ^{2} \pi z} d z=1
$$

and

$$
\frac{1}{2 \pi i} \int_{L} \frac{1}{z+a} \frac{\pi^{2}}{\sin ^{2} \pi z} d z=\psi^{\prime}(a)-\frac{1}{a^{2}} .
$$

Proof. For the first, we just integrate

$$
\begin{aligned}
& \frac{1}{2 \pi i} \int_{L} \frac{\pi^{2}}{\sin ^{2} \pi z} d z=-\frac{1}{2 i}[\cot (\pi z)]_{x_{0}-i y}^{x_{0}+i y} \\
= & \lim _{y \rightarrow \infty}-\frac{1}{2}\left(\frac{\mathrm{e}^{i x_{0}-y}+\mathrm{e}^{-i x_{0}+y}}{\mathrm{e}^{i x_{0}-y}-\mathrm{e}^{-i x_{0}+y}}-\frac{\mathrm{e}^{i x_{0}+y}+\mathrm{e}^{-i x_{0}-i y}}{\mathrm{e}^{i x_{0}+y}-\mathrm{e}^{-i x_{0}-y}}\right)=1 .
\end{aligned}
$$

For the second one, we first integrate twice by parts. This gives

$$
\int_{L} \frac{1}{a+z} \frac{\pi^{2}}{\sin ^{2} \pi z} d z=-2 \int_{L} \frac{\log \sin \pi z}{(a+z)^{3}} d z
$$

Next,we use the product representation for the sine function:

$$
\int_{L} \frac{\log \sin \pi z}{(a+z)^{3}} d z=\int_{L} \frac{\log \pi z}{(a+z)^{3}} d z+\int_{L} \frac{1}{(a+z)^{3}} \sum_{k=1}^{\infty} \log \left(1-\frac{z^{2}}{k^{2}}\right) d z .
$$

The first term gives no contribution, for

$$
\begin{aligned}
\int_{L} \frac{\log \pi z}{(a+z)^{3}} d z & =-\frac{1}{2}\left[\frac{\log \pi z}{\left(a+x_{0}+i y\right)^{2}}\right]_{y=-\infty}^{y=+\infty}+\frac{1}{2} \int_{L} \frac{1}{z(a+z)^{2}} d z \\
& =0+\frac{1}{2}\left[\frac{1}{a(a+z)}-\frac{1}{a^{2}} \log \left(1+\frac{a}{z}\right)\right]_{y=-\infty}^{y=+\infty}=0 .
\end{aligned}
$$

In the second term, due to uniform convergence, we can twist the sum with the integration. We have

$$
\int_{L} \frac{1}{(a+z)^{3}} \log \left(1-\frac{z^{2}}{k^{2}}\right) d z=0-\int_{L} \frac{z}{(a+z)^{2}} \frac{1}{k^{2}-z^{2}} d z .
$$

Assuming $\operatorname{Re}(a)>0$, we can deform the path $L$ to a contour of Hankel type: starting at infinity on the upper side of the real axis, turning around 
the point $z=k$ and going back to infinity below the real axis. Since the integrand vanishes as $z^{-3}$ for large $\operatorname{Re}(z)$, we can further deform the path of integration to a circle around the point $z=k$. This gives

$$
\int_{L} \frac{z}{(a+z)^{2}} \frac{1}{k^{2}-z^{2}} d z=-\frac{\pi i}{(a+k)^{2}}
$$

and hence the second formula of the lemma follows recalling the definition of the digamma function $\psi(z)$, see [41, 8.36].

Now we can use the result of the latter lemma to give an explicit expression for the function $I(z)$.

Lemma 3.4. Let $I(z)$ be the function defined in equation (3.4), then

$$
I(z)=1-2 z+2 \psi^{\prime}(1+z) z^{2} .
$$

Proof. We observe that

$$
\frac{(1-s) s}{(s+z)(s-1+z)}=-1+\frac{z(1+z)}{s+z}+\frac{z(1-z)}{s-1+z},
$$

from which it follows that

$$
\begin{aligned}
\frac{z}{2 \pi i} \int_{L} \frac{(1-s) s}{(s+z)(s-1+z)} \frac{\pi^{2}}{\sin ^{2} \pi s} d s= & -\frac{z}{2 \pi i} \int_{L} \frac{\pi^{2}}{\sin ^{2} \pi s} d s \\
& +\frac{z^{2}(1+z)}{2 \pi i} \int_{L} \frac{1}{s+z} \frac{\pi^{2}}{\sin ^{2} \pi s} d s \\
& +\frac{z^{2}(1-z)}{2 \pi i} \int_{L} \frac{1}{s-1+z} \frac{\pi^{2}}{\sin ^{2} \pi s} d s .
\end{aligned}
$$

Using Lemma 3.3. and recalling that $\psi(z+1)=\psi(z)+\frac{1}{z}$, after some calculation we have the stated formula.

Proposition 3.5. For any $\lambda \in \rho\left(H_{\alpha}\right) \cap \rho\left(H_{0}\right)$, the trace of the relative resolvent of the pair of operators $\left(H_{\alpha}, H_{0}\right)$ is given by

$$
\begin{aligned}
r\left(\lambda ; H_{\alpha}, H_{0}\right) & =-\left.\frac{z I(z)}{\gamma\left(4 \pi \alpha-\gamma F_{\gamma}(z)\right)}\right|_{z=\frac{\gamma}{2 \sqrt{-\lambda}}} \\
& =-\left.\frac{z\left(2 \psi^{\prime}(1+z) z^{2}-2 z+1\right)}{\gamma\left(4 \pi \alpha-\gamma\left(\psi(1+z)-\log z-\frac{1}{2 z}-\psi(1)-\psi(2)\right)\right)}\right|_{z=\frac{\gamma}{2 \sqrt{-\lambda}}},
\end{aligned}
$$

with $\operatorname{Re} \sqrt{-\lambda}>0$. Moreover the following asymptotic expansion holds true for small $\lambda$,

$$
r\left(\lambda ; H_{\alpha}, H_{0}\right)=\sum_{k=0}^{\infty} b_{k}(-\lambda)^{k},
$$


with $b_{k} \in \mathbb{R}$. The first coefficients are given by

$$
b_{0}=-\frac{1}{3 \gamma(\gamma-2 C \gamma+4 \pi \alpha)}, \quad b_{1}=\frac{(17-24 C) \gamma+48 \pi \alpha}{45 \gamma^{3}(2 C \gamma-\gamma-4 \pi \alpha)^{2}} .
$$

For large $\lambda$, we have the following asymptotic expansion

$$
r\left(\lambda ; H_{\alpha}, H_{0}\right)=\sum_{j=2, k=0}^{\infty} a_{j, k}(-\lambda)^{-\frac{j}{2}} \log ^{k}(-\lambda),
$$

with $a_{j, k} \in \mathbb{R}$. The first coefficients are given by

$$
\begin{gathered}
a_{2,0}=-\frac{1}{2}, \quad a_{2, k>0}=0 \\
a_{3,0}=\frac{4 \pi \alpha+(2-C) \gamma+\gamma(\log \gamma-\log 2)}{2}, \quad a_{3,1}=-\frac{\gamma}{4}, \quad a_{3, k>1}=0 .
\end{gathered}
$$

Proof. Formula (3.5) follows directly from the equation (3.3) and from Lemma 3.4. The asymptotic expansions of the relative trace follow easily from classical expansion of the poly Gamma function. Recalling the expansions of the digamma function (see for example [41, 8.344]) for large $|z|, z \in \mathbb{C}$

$$
\psi(1+z)=\log z+\frac{1}{2} \frac{1}{z}-\sum_{k=1}^{\infty} \frac{B_{2 k}}{2 k} \frac{1}{z^{2 k}},
$$

$B_{2 k}$ being Bernoulli numbers, and for small $z \in \mathbb{C}$ (see for example 41, 8.342]):

$$
\psi(1+z)=-C+\sum_{k=2}^{\infty}(-1)^{k} \zeta(k) z^{k-1},
$$

where $C$ is the Euler constant, and where $\zeta$ denotes the Riemann's zeta function. Since $z=\frac{\gamma}{2 \sqrt{-\lambda}}$ one obtains the expansions (3.6) and (3.7).

\section{The Relative partition function of the Coulomb Plus delta INTERACTION}

In this section we study the relative zeta function and the relative partition function of the model described in Section 3 .

In order to obtain the relative zeta function for the pair of operators $\left(H_{\alpha}, H_{0}\right)$ described in Section 3. It is clear, by the result in Prop. 3.5, that the conditions (B.1), (B.2) and (B.3) of Lemma 2.1, necessary to define the relative zeta function are satisfied. Also, by the same proposition, the minimum value for the index $j$ is $j=2$, corresponding to $\alpha_{2}=-1$, then the first terms in the expansion of the relative spectral measure, according to Lemma 2.4, are: first, the term corresponding to $\alpha_{2}=-1$, that gives a only a term in $\frac{1}{v}$, since $K_{2}=0$; second, the terms corresponding to $\alpha_{3}=-\frac{3}{2}$, 
that gives a term in $\frac{1}{v^{2}}$ and a term in $\frac{1}{v^{2}} \log v^{2}$, since $K_{3}=1$. Applying the formula in Lemma 2.4, the coefficients are:

$$
\begin{aligned}
& e_{2,0,0}=0 \\
& e_{3,0,0}=\frac{4 \pi \alpha+(2-C) \gamma+\gamma \log \frac{\gamma}{2}}{\pi}, \quad e_{3,1,0}=0, \quad e_{3,1,1}=-\frac{\gamma}{2 \pi} .
\end{aligned}
$$

Whence, we have the following expansion of the relative spectral measure:

$$
e\left(v ; H_{\alpha}, H_{0}\right)=O\left(v^{k}\right), \quad k>0
$$

for $v \rightarrow 0^{+}$,

$$
e\left(v ; H_{\alpha}, H_{0}\right)=-\frac{\gamma}{\pi} \frac{1}{v^{2}} \log v+\frac{4 \pi \alpha+(2-C) \gamma+\gamma \log \frac{\gamma}{2}}{v^{2}}+O\left(v^{-3} \log v\right)
$$

for $v \rightarrow+\infty$, and $e_{3,0}=\frac{4 \pi \alpha+(2-C) \gamma+\gamma \log \frac{\gamma}{2}}{\pi}, e_{3,1}=-\frac{\gamma}{\pi}$. All the coefficients $e_{j, h}$ with smaller indices vanish.

We are now in the position of analyzing the relative zeta function $\zeta\left(s ; H_{\alpha}, H_{0}\right)$. In fact, what we are interested in is the expansion near $s=-\frac{1}{2}$.

Proposition 4.1. The relative zeta function $\zeta\left(s ; H_{\alpha}, H_{0}\right)$ has an analytic expansion to a meromorphic function analytic in the strip $0 \leq \operatorname{Re}(s) \leq 1$, up to a double pole at $s=-\frac{1}{2}$. Near $s=-\frac{1}{2}$, the following expansion holds:

$$
\begin{aligned}
\zeta\left(s ; H_{\alpha}, H_{0}\right)= & \frac{e_{3,1} / 4}{\left(s+\frac{1}{2}\right)^{2}}+\frac{e_{3,0} / 2}{s+\frac{1}{2}}+\int_{0}^{1} v e\left(v ; H_{\alpha}, H_{0}\right) d v \\
& +\int_{1}^{\infty} v\left(e\left(v ; H_{\alpha}, H_{0}\right)-\frac{e_{2,1}}{v^{2}} \log v-\frac{e_{2,0}}{v^{2}}\right) d v+O\left(s+\frac{1}{2}\right),
\end{aligned}
$$

where

$$
e_{3,1}=-\frac{\gamma}{\pi}, \quad e_{3,0}=\frac{8 \pi \alpha-2 C \gamma+4 \gamma+\gamma \log \frac{\gamma^{2}}{4}}{2 \pi} .
$$

Proof. By the expansions in equations (4.1) and (4.2) for the relative spectral measure, we see that the indices $J_{0}$ and $J_{\infty}$ defined in Lemma 2.7 are respectively: $J_{0}=0$ and $J_{\infty}=4$. Hence, by the same lemma, there are no poles arising from the expansion of the spectral measure for small $v$, and since the minimum value for the index $j$ of $\alpha_{j}$ is $j=2$, there are three terms arising from the expansion for large $v$. The first term is with $j=2$, and vanishes since $e_{2,0}=0$. The other two terms are with $j=3$, and $k=0$ and $k=H_{3}=1$. Applying the formula in Lemma 2.7 we compute these terms.

Corollary 4.2. The relative zeta function of the pair of operators $(L=$ $\left.-\partial_{u}^{2}+H_{\alpha}, L_{0}=-\partial_{u}^{2}+H_{0}\right)$ on $S_{\frac{\beta}{2 \pi}}^{1} \times \mathbb{R}^{3}$ has a simple pole at $s=0$ with 
residua:

$$
\begin{aligned}
& \operatorname{Res}_{s=0} \zeta\left(s ; L, L_{0}\right)=\frac{\gamma}{4 \pi} \beta, \\
& \operatorname{Res}_{s=0} \zeta\left(s ; L, L_{0}\right)=-\frac{8 \pi \alpha-2 C \gamma+4 \gamma+\gamma \log \frac{\gamma^{2}}{4}}{4 \pi} \beta+\frac{(1-\log 2) \gamma}{2 \pi} \beta, \\
& \operatorname{Res}_{\substack{s=0 \\
s=}} \zeta^{\prime}\left(s ; L, L_{0}\right) \\
= & -\left(\int_{0}^{1} v e\left(v ; H_{\alpha}, H_{0}\right) d v+\int_{1}^{\infty} v\left(e\left(v ; H_{\alpha}, H_{0}\right)-\frac{e_{3,1}}{v^{2}} \log v-\frac{e_{3,0}}{v^{2}}\right) d v\right) \beta \\
& -\frac{(1-\log 2)\left(8 \pi \alpha-2 C \gamma+4 \gamma+\gamma \log \frac{\gamma^{2}}{4}\right)}{2 \pi} \beta+\left(2+\frac{\pi^{2}}{6}+2(1-\log 2)^{2}\right) \frac{\gamma}{4 \pi} \beta \\
& -2 \int_{0}^{\infty} \log \left(1-\mathrm{e}^{-\beta v}\right) e\left(v ; H_{\alpha}, H_{0}\right) d v .
\end{aligned}
$$

Proof. This is a simple consequence of Prop. 4.1 and Cor. 2.8 .

Using the formula in equation (2.3), we obtain the following result for the relative partition function, where $\ell$ is some renormalization constant,

$$
\begin{aligned}
& \log Z_{\mathrm{R}} \\
= & -\frac{1}{2}\left(\int_{0}^{1} v e\left(v ; H_{\alpha}, H_{0}\right) d v+\int_{1}^{\infty} v\left(e\left(v ; H_{\alpha}, H_{0}\right)-\frac{e_{3,1}}{v^{2}} \log v-\frac{e_{3,0}}{v^{2}}\right) d v\right) \beta \\
& -\frac{(1-\log 2)\left(8 \pi \alpha-2 C \gamma+4 \gamma+\gamma \log \frac{\gamma^{2}}{4}\right)}{4 \pi} \beta+\left(2+\frac{\pi^{2}}{6}+2(1-\log 2)^{2}\right) \frac{\gamma}{8 \pi} \beta \\
& -\int_{0}^{\infty} \log \left(1-\mathrm{e}^{-\beta v}\right) e\left(v ; H_{\alpha}, H_{0}\right) d v \\
& +\left(4 \pi \alpha-C \gamma+2 \gamma+\gamma \log \frac{\gamma}{2}-1+\gamma \log 2\right) \frac{\beta}{2 \pi} \log \ell .
\end{aligned}
$$

Acknowledgments. The authors are very pleased to dedicate this work to Pavel Exner, on the occasion of his 70th birthday. He has always been for us a source of inspiration, and we are very grateful to him for his support. We thank the referee for the very useful suggestions and for pointing out additional references. The authors acknowledge the hospitality and financial support of the CIRM, University of Trento, Italy. C.C. also gratefully acknowledges the hospitality of the Mathematical Institute, Tohoku University, Sendai, Japan, of the Hausdorff Center for Mathematics, University of Bonn, Germany, and the support of the FIR 2013 project, code RBFR13WAET. 


\section{RELATIVE PARTITION FUNCTION OF COULOMB PLUS DELTA INTERACTION 21}

\section{REFERENCES}

[1] S. Albeverio, Wiener and Feynman-path integrals and their applications, Proceedings of the Norbert Wiener centenary congress, AMS Press, 1997.

[2] S. Albeverio, Z. Brzeźniak, and L. Dabrowski, Fundamental solution of the heat and Schrödinger equations with point interaction, J. Funct. Anal. 130 (1995), 220-254.

[3] S. Albeverio, G. Cognola, M. Spreafico, and S. Zerbini, Singular perturbations with boundary conditions and the Casimir effect in the half space, J. Math. Phys. 51 (2010), 063502, 38pp.

[4] S. Albeverio, F. Gesztesy, R. Høegh-Krohn, and H. Holden, Solvable models in quantum mechanics: Second edition, AMS Chelsea Publ., 2005, with an Appendix by P. Exner.

[5] S. Albeverio, P. Kurasov, Singular perturbations of differential operators, Cambridge University Press (2000).

[6] M. Asorey, A. Ibort, and G. Marmo, Global theory of quantum boundary conditions and topology change, Int. J. Mod. Phys. A 20 (2005), 1001-1026.

[7] M.F. Atiyah, V.K. Patodi, and I.M. Singer, Spectral asymmetry and Riemannian geometry, III, Math. Proc. Cambridge Phil. Soc. 79 (1976), 71-99.

[8] E. Beth, G.E. Uhlenbeck, The quantum theory of the non-ideal gas. II. Behaviour at low temperatures, Physica 4 (1937), 915-924.

[9] M.Š. Birman, M. G. Krein, On the theory of wave operators and scattering operators, Soviet Math. Dokl 3 (1962), 740-744.

[10] L. Boi, The Quantum Vacuum, The J. Hopkins Univ. Press, Baltimore (2011).

[11] M. Bordag, ed., The Casimir effect 50 years later, in "Proceedings the IV Workshop on quantum field theory under the influence of external conditions", Leipzig, 1998, World Scient., Singapore (1999).

[12] M. Bordag, G.L. Klimchitskaya, U. Mohideen, and V.M. Mostepanenko, Advances in the Casimir effect, Oxford UP (2009).

[13] M. Bordag, V.V. Nesterenko, and I.G. Pirozhenko, High temperature asymptotics of thermodynamic functions of an electromagnetic field subjected to boundary conditions on a sphere and cylinder, Physical Review D 65 (2002), no. 4, 045011.

[14] M. Bordag, I.G. Pirozhenko, and V.V. Nesterenko, Spectral analysis of a flat plasma sheet model, J. Phys. A 38 (2005), 11027.

[15] M. Bordag, D.V. Vassilevich, Heat kernel expansion for semitransparent boundaries, J. Phys. A 32 (1999), 8247-8259.

[16] J. Brüning, V. Geyler, and K. Pankrashkin, On-diagonal singularities of the Green functions for Schrödinger operators, J. Math. Phys. 46 (2005), 113508, 16pp.

[17] J. Brüning, R. Seeley, The resolvent expansion for second-order regular singular operators, J. Funct. Analysis 73 (1987), 369-429.

[18] G. Bressi, G. Carugno, R. Onofrio, and G. Ruoso, Measurement of the Casimir force between parallel metallic plates, Phys. Rev. Lett. 88 (2002).

[19] A.A. Bytsenko, E. Elizalde, S. Odintsov, A. Romeo, and S. Zerbini, Zeta-function regularization with applications, World Scientific (1994).

[20] H.B.G. Casimir, On the attraction between two perfectly conducting plates, Proceedings of the Royal Netherlands Academy of Arts and Sciences 51 (1948), 793-795.

[21] H.G.B. Casimir, On the attraction of two perfectly conducting plates, Proc. Kongl. Nedel. Akad. Wetensch 51 (1948), 793-795.

[22] J. Choi, J.R. Quine, Zeta regularized products and functional determinants on spheres, Rocky Mt. J. Math. 26 (1996), 719-729. 
[23] G. Cognola, L. Vanzo, and S. Zerbini Vacuum energy in arbitrarily shaped cavities J. Math. Phys. 33 (1992), 222-228.

[24] Y. Colin de Verdière, Pseudo-Laplaciens II, Ann. Inst. Fourier, Grenoble 33 (1983), $87-113$.

[25] M.V. Cougo Pinto, C. Farina, M.R. Negrão, and A. Tort, Casimir effect at finite temperature of charged scalar field in an external magnetic field, arXiv:hep-th/9810033 (1998), 6pp.

[26] J.S. Dowker, G. Kennedy, Finite temperature and boundary effects in static spacetimes, J. Phys. A 11 (1978), 895.

[27] J.P. Dowling, The mathematics of the Casimir effect, Math. Mag. 62 (1989), 324331.

[28] G.V. Dunne, K. Kirsten, Simplified vacuum energy expressions for radial backgrounds and domain walls, J. Phys. A: Math. and Theo. 42 (2009), no. 7, 075402.

[29] E. Elizalde, Ten physical applications of spectral zeta functions, Springer, Berlin (1995).

[30] E. Elizalde, S.D. Odintsov, A. Romeo, A.B. Bytsenko, and S. Zerbini, Zeta Regularization Techniques and Applications, World Scient., Singapore (1999).

[31] E. Elizalde, L. Vanzo, and S. Zerbini, Zeta-function regularization, the multiplicative anomaly and the Wodzicki Residue, Comm. Math. Phys. 194 (1998), 613-630.

[32] A. Erdélyi, et al., eds. Tables of Integral Transforms: Vol.: 2. McGraw-Hill, 1954.

[33] D. Fermi, L. Pizzocchero, Local zeta regularization and the Casimir effect, Progr. Theor. Phys. 126 (2011), 419-434.

[34] D. Fermi, L. Pizzocchero, Local zeta regularization and the Casimir effect I-IV, arXiv:1505.00711, 1505.01044, 1505.01651, 1505.03276 [math-ph] (2015).

[35] M. Fierz, On the attraction of conducting planes in vacuum, Helv. Phys. Acta 33 (1960), 855

[36] R. Figari, R. Höegh-Krohn, and C.R. Nappi, Interacting relativistic boson fields in the de Sitter universe with two space-time dimensions Comm. Math. Phys. 44 (1975), $265-278$.

[37] S.A. Fulling, Aspects of quantum field theory in curved space-time, Cambridge Univ. Press (1989).

[38] G. Gibbons, Thermal zeta functions, Phys. Letters A 60 (1977), 385-386.

[39] P.B. Gilkey, Invariance theory, the heat equation, and the Atiyah-Singer index theorem, Studies in Advanced Mathematics (CRC Press, 1995).

[40] P.B. Gilkey, K. Kirsten, and D.V. Vassilevich, Heat trace asymptotics defined by transfer boundary conditions, Lett. Math. Phys. 63 (2003), 29-37.

[41] I. S. Gradshteyn and I. M. Ryzhik, Table of integrals, series, and products. $7^{\text {th }}$ ed., Academic Press, 2007.

[42] N. Graham, R.L. Jaffe, V. Khemani, M. Quandt, M. Scandurra, and H. Weigel, Calculating vacuum energies in renormalizable quantum field theories: $A$ new approach to the Casimir problem, Nucl. Phys. B 645 (2002), 49-84.

[43] S.W. Hawking, Zeta function regularization of path integral in curved spacetime, Comm. Math. Phys. 55 (1977), 133-148.

[44] L. Hostler, Runge-Lenz vector and the Coulomb Green's functions, J. Math. Phys. 8 (1967), no. 3, 642-646.

[45] N.R. Khusnutdinov, Zeta-function approach to Casimir energy with singular potentials, Phys. Rev. D 73 (2006), 025003.

[46] M.G. Krein, On the trace formula in perturbation theory, Mat. Sb. (N.S.) 33 (1953), no. $75,597-626$. 
[47] M.G. Krein, Perturbation determinants and a formula for the traces of unitary and self-adjoint operators, Sov. Math. Dokl 3 (1962), 707-710

[48] N. Kurokawa, Multiple sine functions and Selberg zeta functions, Proceedings of the Japan Academy, Series A, Mathematical Sciences 67 (1991), 61-64.

[49] S.K. Lamoureaux, Demonstration of the Casimir force in the 0.6 to $6 \mu m$ range, Phys. Rev. Lett. 78 (1997), 5-8.

[50] M. Lesch, Determinants of regular singular Sturm-Liouville operators, Math. Nachr. 194 (1998), 139-170.

[51] J. Mehra, Temperature correction to the Casimir effect, Physica 37 (1967), no. 1, $145-152$.

[52] K.A. Milton, The Casimir effect: The Physical Manifestation of Zero-Point Energy, World Scientific, Singapore (2001).

[53] V. Moretti, Local $\zeta$-function techniques vs. point-splitting procedure: a few rigorous results, Comm. Math. Phys. 201 (1999), 327-363.

[54] V.M. Mostepanenko, N.N. Trunov, The Casimir Effect and its Applications, Oxford, Clarendon Press (1997).

[55] W. Müller, Relative zeta functions, relative determinants and scattering theory, Comm. Math. Phys. 192 (1998), 309-347.

[56] J.M. Muñoz Castañeda, J. Mateos Guilarte, $\delta-\delta^{\prime}$ generalized Robin boundary conditions and quantum vacuum fluctuations, Phys. Rev. D 91 (2015), 025028, 21pp.

[57] V.V. Nesterenko, G. Lambiase, and G. Scarpetta, Calculation of the Casimir energy at zero and finite temperature: Some recent results, Riv. Nuovo Cimento 27 (2004), no.6, $1-74$.

[58] V.V. Nesterenko, I.G. Pirozhenko, Lifshitz formula by a spectral summation method, Phys. Rev. A 86 (2012), 052503.

[59] G. Ortenzi, M. Spreafico, Zeta function regularization for a scalar field in a bounded domain, J. Phys. A 37 (2004), 11499-11517.

[60] D.K. Park, Green's function approach to two and three-dimensional delta function potentials and application to the spin 1/2 Aharonov-Bohm problem, J. Math. Phys. 36 (1995), 5453-5464.

[61] G. Plunien, B. Müller, and W. Greiner, The Casimir effect, Phys. Repts. 134 (1986), 87-193.

[62] E.A. Power, Introductory Quantum Electrodynamics, Longman, London (1964).

[63] D.B. Ray, I.M. Singer, R-torsion and the Laplacian on Riemannian manifolds, Adv. Math. 7 (1974), 145-210.

[64] M. Scandurra, The ground state energy of a massive scalar field in the background of a semi-transparent spherical shell, J. Phys. A 32 (1999), 5679.

[65] S. Scarlatti, A. Teta, Derivation of the time dependent propagator for the threedimensional Schrödinger equation with one point interaction, J. Phys. A 23 (1990), L1033-L1035.

[66] S.N. Solodukhin, Exact solution for a quantum field with delta like interaction, Nucl. Phys. B 541 (1999), 461-482.

[67] M.J. Spaarnay Measurements of attractive forces between flat plates, Physica 24 (1958), 751.

[68] M. Spreafico, Zeta function and regularized determinant on projective spaces, Rocky Mt. J. Math. 33 (2003), 1499-1512.

[69] M. Spreafico, A generalization of the Euler Gamma function, Funct. Analysis Applic. 39 (2005), 87-91. 
[70] M. Spreafico, Zeta invariants for sequences of spectral type, special functions and the Lerch formula. Proceedings of the Royal Society of Edinburgh: Section A Mathematics 136, no. 4, (2006) 863-887.

[71] M. Spreafico, Zeta determinant and operator determinants, Osaka Jour. Math. 48 (2011), 41-50.

[72] M. Spreafico, S. Zerbini, Finite temperature quantum field theory of non compact domain and application to delta interaction, Rep. Math. Phys. 63 (2009), 163-177.

[73] K. Symanzik, Schrödinger representation and Casimir effect in renormalizable quantum field theory, Nuclear Phys. B 190 (1981), no. 1, 1-44.

Institut für Angewandte Mathematik, Universität Bonn, Endenicher Allee 60, 53115 Bonn, Germany; SFB611, Bonn; HCM, Bonn; BIBOS, Bielefeld and Bonn; IZKS, Bonn; CERFIM, Locarno; Acc. Arch., USi, Mendrisio; Dip. Matematica Università di Trento, Italy

E-mail address: albeverio@uni-bonn.de

Dipartimento di Scienza e Alta Tecnologia, Università dell'Insubria, Via VAlleggio 11, 22100 Como, Italy

E-mail address: claudio.cacciapuoti@uninsubria.it

Dipartimento di Matematica e Fisica Ennio De Giorgi, Università del Salento \& INFN, Via Arnesano, 73100 Lecce, Italy

E-mail address: mauro.spreafico@unisalento.it 